\section{PRODUCCIÓN CIENTÍFICA SOBRE FÚTBOL EN REVISTAS ESPAÑOLAS DE CIENCIAS DE LA ACTIVIDAD FÍSICA Y DEL DEPORTE}

\author{
PRODUÇÃO CIENTÍFICA SOBRE FUTEBOL EM REVISTAS ESPANHOLAS \\ DE CIÊNCIAS DA ATIVIDADE FÍSICA E ESPORTES
}

\author{
SCIENTIFIC PRODUCTION ON FOOTBALL IN SPANISH JOURNALS OF \\ PHYSICAL ACTIVITY AND SPORT SCIENCES
}

\section{Xabier Martín-Nebreda*, Mikel Pérez-Gutiérrez **, María Teresa Gómez-Alonso*, Carlos Gutiérrez-García*}

Palabras-clave

Fútbol.

Bibliometría.

Actividades

científicas.

Artículo de revista.

Deportes.
Resumen: Este estudio analiza, desde un punto de vista bibliométrico, los artículos sobre fútbol publicados en 40 revistas españolas incluidas en la categoría de "Deportes" del Catálogo Latindex, entre los años 2001 y 2012. Los aspectos analizados fueron: (1) número de artículos publicados sobre fútbol, porcentaje respecto al total de artículos, y distribución según revistas, (2) evolución temporal, y (3) disciplinas presentes. Los resultados muestran que la producción científica sobre fútbol ha sido relevante $(7,9 \%$ del total de artículos publicados), creciente, multidisciplinar y dispar entre las disciplinas y revistas, destacando las revistas y disciplinas de psicología, legislación y organización y gestión.

Resumo: Este estudo analisa, do ponto de vista bibliométrico, os artigos sobre futebol publicados em 40 revistas espanholas incluídas na categoria "Esportes", do Catálogo Latindex, entre os anos 2001 e 2012. Os aspetos analisados foram: (1) O número de artigos publicados sobre o futebol, percentagem relativa ao total de artigos e distribuição segundo as revistas; (2) Evolução temporal; (3) Disciplinas presentes. Os resultados mostram que a produção científica sobre o futebol foi relevante $(7,9 \%$ do total de artigos publicados), crescente, multidisciplinar e díspar entre as disciplinas e revistas, destacando-se as revistas e disciplinas de psicologia, de legislação, de organização e de gestão.

Abstract: study analyzes, from a bibliometric perspective, papers published in 40 Spanish journals under the category "deportes" (sports) of the Latindex Catalogue, between 2001 and 2012. Aspects analyzed include: (1) number of papers published about football; percentage regarding total papers published; and distribution among journals; (2) evolution over time; and (3) disciplines. Results showed that the scientific production on football was prominent (7.9\% of total published papers), on the rise, multidisciplinary, and unequal among journals and disciplines. The most outstanding disciplines were those related to Psychology, Law, Organization, and Management.
*Universidad de León. León, Espanha. E-mail: xmartn00@estudiantes.unileon.es

**Universidad Autónoma de Chile. Santiago, Chile.

E-mail: mikel.perez@uautonoma.cl

Recebido em: 18-03-2015

Aprovado em: 07-06-2015

(c) (i) () Licence 


\section{INTRODUCCIÓN}

El fútbol es una de las prácticas sociales más populares de nuestro tiempo (TIESLER; COELHO, 2008). Forma parte de la vida del ciudadano y, le guste o no, convive con él diariamente en la radio, televisión, periódicos y en conversaciones propias 0 ajenas. Es posiblemente el deporte más practicado del mundo. Según la FIFA (2007), más de 265 mil personas de ambos sexos juegan al fútbol en sus 207 federaciones nacionales asociadas, y más de 270 mil están implicadas en su desarrollo, incluyendo a técnicos, asistentes y árbitros. Según recogen Navarro-García, Reyes-García y Acedo-González (2014), el fútbol es en datos financieros la $17^{a}$ economía más importante del mundo, con ventas de 377 mil milliones de euros. El Mundial de Brasil 2014, de acuerdo con los datos aportados por la FIFA (2014), recibió una afluencia de casi 3,5 miliones de espectadores en los 64 partidos disputados, acreditó a más de 16 mil trabajadores de medios de comunicación, y hasta 214 países recibieron la señal de televisión del evento.

En España, el fútbol es sin duda el deporte rey. Según los datos del Consejo Superior de Deportes (CSD), ocupa el primer lugar como líder absoluto en cuanto a número de licencias federativas, con algo más de 850 mil afiliados, seguido del baloncesto con 400 mil, y la caza con 350 mil (CONSEJO SUPERIOR DE DEPORTES, 2014). Por su parte, la última encuesta de hábitos deportivos de los españoles (periodo 2005-2010) sitúa al fútbol como la segunda actividades físico-deportivas más practicada, con un $24,6 \%$ de las personas que practicaban alguna actividad físico-deportiva, solo por detrás de la gimnasia y actividades físicas guiadas, con un 34,6\% (GARCÍA; LLOPIS, 2011). Asimismo, en su vertiente de espectáculo de masas, el fútbol es un fenómeno social cuya importancia trasciende largamente el ámbito estrictamente deportivo. Empresas e instituciones buscan el patrocinio deportivo de clubes de fútbol como mejora de su imagen corporativa, los dirigentes políticos anhelan imágenes con héroes deportivos para el aumento de su popularidad y los medios de comunicación utilizan el fútbol como uno de sus principales contenidos para mejorar sus ingresos e índices de audiencia (GARCÍA; LLOPIS, 2011).

La importancia del fútbol como fenómeno social se refleja también en el ámbito académico. Forma parte de las enseñanzas deportivas, orientadas a formar técnicos deportivos, en este caso de fútbol. También está presente las titulaciones universitarias de Grado en Ciencias de la Actividad Fisca y del Deporte (en adelante CCAFD), donde se da cabida a asignaturas específicas como "Fundamentos de Fútbol" o "Ampliación deportiva Fútbol". A nivel de máster, existen diversos títulos específicos como el "Máster Universitario en Preparación Física en Fútbol" (Universidad de Castilla La Mancha y Real Federación Española de Fútbol), o el "Máster en Rendimiento Deportivo: Tecnificación y Alto Nivel con especialidad en Fútbol" (Instituto Nacional de Educación Física de Cataluña), entre otros.

En lo que se refiere a la investigación, el fútbol es objeto permanente de análisis desde las CCAFD. Su carácter multidisciplinar integra a investigadores de diversos campos de conocimiento, y se muestra fundamentalmente a través de artículos científicos publicados en revistas genéricas del ámbito de las CCAFD o específicas de fútbol. En un ámbito internacional pueden destacarse publicaciones específicas como Soccer \& Society o Football Science, 0 la revista genérica Journal of Sport Sciences, que incorpora regularmente un suplemento sobre ciencia y medicina en fútbol. En España no existe ninguna revista científica sobre fútbol, 
aunque sí revistas técnicas como abfutbol, Fútbol-táctico, Training Fútbol, Cuaderno de Fútbol, o FutbolPF. Esto no ha impedido que desde el conjunto de revistas españolas de CCAFD se haya generado ya una abundante literatura científica en torno a este deporte.

\section{ANTECEDENTES}

La producción científica existente sobre el fútbol hoy en día es muy abundante. A modo de ejemplo, la búsqueda combinada de los términos "soccer" y "football" en la colección principal de la Web of Science muestra 15.431 artículos y revisiones publicados desde 1990 (consulta realizada el 27 de enero de 2015). Sin embargo, hasta donde sabemos no existe ningún estudio bibliométrico que evalúe de forma general la producción científica sobre fútbol a nivel internacional ni tampoco en el contexto español. Ello, a pesar de la indudable utilidad de los estudios bibliométricos como medio para conocer y valorar el desarrollo de la ciencia en general y de cualquiera de sus disciplinas y campos en particular (USING..., 2008), así como para comparar la producción científica realizada en diversos países, instituciones o por grupos de investigación o investigadores concretos.

De hecho, las CCAFD han mostrado tradicionalmente un menor interés por la bibliometría que otras ciencias como la pedagogía, sociología, psicología o medicina (LIDOR; MILLER; MAHARIK, 2002). No obstante, durante los últimos años la creciente importancia y popularidad de los estudios bibliométricos (KAUR; RADICCHI; MENCZER, 2013) así como la plena consolidación y desarrollo de las CCAFD en el ámbito académico (BIRRELL, 2006) ha dado como resultado la publicación de diversos trabajos de aplicación bibliométrica. Entre los mismos, sobre la presencia de las CCAFD en bases de datos específicas (GAU, 2013), ámbitos del deporte como la economía, la gestión deportiva o la religión (CIOMAGA, 2013, PÉREZ-GUTIÉRREZ et al., 2015, SÁNCHEZ; CASTELLANOS, 2011), revistas específicas (NEVILL; ATKINSON; HUGHES, 2008), sus índices de impacto (MCBRIDE, 2006), o deportes concretos (MARTíN et al., 2013; PÉREZ-GUTIÉRREZ; GUTIÉRREZ-GARCÍA, 2009; PESET et al., 2013), son algunos ejemplos de ámbito internacional.

El caso español ha seguido la misma tendencia que el contexto internacional. Aunque existen antecedentes (PIERNAVIEJA, 1965), no ha sido hasta principios del presente siglo cuando los estudios bibliométricos en CCAFD han logrado una mayor presencia. Mención expresa merecen los trabajos desarrollados por Devís, Valenciano, Villamón y colaboradores en relación a las revistas científicas españolas de CCAFD (véanse e.g., DEVÍS et al., 2003; 2010; VALENCIANO et al., 2009; 2010; VILLAMÓN; DEVÍS; VALENCIANO, 2005). Desde otras perspectivas, se han realizado estudios sobre campos tan diversos como la psicología del deporte (SALVADOR et al., 2007), cardiología y deporte (MARTÍNEZ et al., 2012), economía y deporte (SÁNCHEZ; CASTELLANOS, 2012), balonmano (GUTIÉRREZ, 2014), rugby (VILLAREJO; PALAO; ORTEGA, 2010), artes marciales (PÉREZ-GUTIÉRREZ; GUTIÉRREZGARCÍA, 2008), o revistas concretas (ARBINAGA; ARAGÓN; TEJEDOR, 2010, LÓPEZ; VELASCO, 2004). Particularmente sobre fútbol pueden citarse los trabajos de Martínez et al. (2002), sobre bibliometría de arbitraje en fútbol, y Fernández-Revelles (2014), que correlacionó la producción científica en fútbol con el ranking FIFA de selecciones nacionales. En síntesis, cada vez son más los investigadores de las CCAFD que utilizan técnicas bibliométricas para el conocimiento, análisis e interpretación de sus ámbitos de especialización. 


\section{MÉTODO}

\subsection{Objetivo y delimitación del estudio}

Como se ha señalado, no existe hasta la fecha ningún estudio bibliométrico genérico sobre fútbol, a pesar de la gran popularidad de este deporte y de la considerable producción científica generada en torno al mismo. A partir de estas consideraciones, el objetivo del presente estudio fue analizar, desde una perspectiva bibliométrica, los artículos originales sobre fútbol publicados en las revistas españolas incluidas en la subdivisión del campo de Ciencias Sociales, tema "Deportes", del Catálogo Latindex, entre los años 2001 y 2012. Este análisis estuvo centrado en los siguientes aspectos: (1) Número de artículos de fútbol, porcentaje respecto al total de artículos publicados, y su distribución según revistas, (2) Evolución temporal, y (3) Disciplinas presentes. Sobre esta definición es preciso realizar una serie de consideraciones:

1) Únicamente fueron objeto de estudio los artículos originales. No fueron objeto de estudio artículos no originales (e.g., traducciones), ni otro tipo de contenidos tales como editoriales, reseñas de libros y tesis, informes sobre eventos científicos, etc.

2) Se consideraron "artículos sobre fútbol" los que, tras un proceso de categorización inductiva a partir del título, resumen y palabras clave, se verificó que se centraban en el fútbol, que la muestra utilizada fue de fútbol, o se realizaba en ellos un estudio con varios deportes, figurando entre ellos el fútbol. Este mismo criterio ya ha sido seguido en investigaciones similares (DEVÍS et al., 2010; VILLAREJO; PALAO; ORTEGA, 2010).

2) Se tomó como referencia Latindex debido a que es un sistema de información internacional (ámbito Latinoamericano) constantemente actualizado, y el Catálogo, en lugar del Directorio, debido a su carácter más selectivo, ya que únicamente las revistas que cumplen con una serie de requisitos mínimos de calidad editorial pasan a formar parte del mismo. Se descartaron sistemas de información españoles como RESH (Revistas Españolas de Ciencias Sociales y Humanidades) o In-Recs por no tener carácter internacional, no estar actualizados, y en el caso de In-Recs por carecer de un apartado específico para las CCAFD. También se descartaron sistemas de información internacionales más selectivos, como las bases de datos de la Web of Science, Scopus o Scielo, debido a la aún escasa presencia de revistas españolas de CCAFD en los mismos.

\subsection{Fuentes de información}

De las 94 revistas incluidas en el tema "Deportes" del Catálogo Latindex, a fecha 11 de noviembre de 2013, se seleccionaron aquellas editadas en España $(n=44)$. Una vez analizada la ficha de cada revista, se decidió unificar tres títulos (Ágora para la educación física y el deporte, Retos, y Revista de Artes Marciales Asiáticas), al tener entradas diferentes para sus ediciones en papel y online o ser continuaciones de un título anterior. Asimismo, no fue posible realizar el análisis de la revista digital The International Journal of Medicine and Science in Physical Education and Sport (2005-2009) debido a que sus artículos ya no están disponibles. 
Finalmente, el número total de revistas analizadas ascendió a 40, cuyos títulos pueden verse más adelante en la Tabla 1.

\subsection{Instrumentos de recogida de información}

Se elaboró una base de datos de artículos de fútbol en el programa gestor de referencias EndNote (v.X6). Además de los campos relativos a la referencia bibliográfica (Autores, año, título del artículo, etc.), se añadió un campo para reflejar la disciplina académica a la que se adscribió cada artículo. El listado de disciplinas partió del utilizado por Devís et al. (2010), al que se añadieron las disciplinas "Reglamento de competición", "Técnica" y "Táctica y estrategia" al ser estas elementos clásicos y fundamentales en el estudio de los deportes.

Una vez completada, esta base de datos se exportó a una hoja de cálculo Microsoft Excel (v.2010) para realizar los cálculos correspondientes. De forma paralela, se elaboró otra hoja de cálculo en la que se registraron los datos básicos de cada revista (título, ISSN, enlace a versión electrónica y a ficha Latindex, año de comienzo y fin) y el número total de artículos y de artículos de fútbol publicados por año y en el conjunto del periodo estudiado.

\subsection{Procedimiento de recogida de información}

La recogida de datos se realizó a través de la consulta directa de las revistas en sus versiones electrónicas. Cuando esto no fue posible, se consultaron los ejemplares impresos. Una vez completado el listado de referencias se realizó el proceso de categorización, asignando a cada artículo su correspondiente disciplina. A cada artículo se le asignó una única disciplina, ya que aunque algunos trabajos podrían abordar varias dicho procedimiento evita posibles problemas de sobre representación (véase en el mismo sentido el trabajo de DEVíS et al., 2010).

\subsection{Análisis y presentación de los datos}

Se realizó una estadística descriptiva basada en el análisis de frecuencias y porcentajes de los resultados obtenidos. Los resultados se muestran en tablas para su mejor comprensión.

\section{RESULTADOS}

El número total de artículos publicados en las revistas analizadas ascendió a 6.371, siendo 502 sobre fútbol (7,9\%). De forma destacada, la revista con mayor producción fue Revista Aranzadi de Derecho del Deporte y Entretenimiento, seguida de la Revista de psicología del deporte y Cuadernos de psicología del Deporte. Por el contrario, un total de 24 revistas (60\% del total) publicaron un promedio de menos de un artículo sobre fútbol por año (Tabla 1). 
Tabla 1 - Número de artículos de fútbol y porcentaje respecto al número total de artículos publicados por revista

\begin{tabular}{|c|c|c|c|c|}
\hline Título & $\begin{array}{l}\text { Comienzo- } \\
\text { Fin }\end{array}$ & $\begin{array}{l}\text { Artículos } \\
\text { fútbol }\end{array}$ & $\begin{array}{c}\text { Artículos } \\
\text { publicados }\end{array}$ & $\%$ \\
\hline Revista Aranzadi de derecho de deporte y entretenimiento & $2001-$ & 100 & 613 & $16,3 \%$ \\
\hline Revista de psicología del deporte & $1992-$ & 39 & 254 & $15,4 \%$ \\
\hline Cuadernos de psicología del deporte & 2001- & 36 & 225 & $16 \%$ \\
\hline Apunts. Educación Física y Deportes & 1993- & 32 & 489 & $6,5 \%$ \\
\hline Kronos & $2002-$ & 27 & 203 & $13,3 \%$ \\
\hline Red. Revista de entrenamiento deportivo & $1981-$ & 27 & 232 & $11,6 \%$ \\
\hline Motricidad. European Journal of Human Movement & $1995-$ & 25 & 244 & $10,2 \%$ \\
\hline Revista española de derecho deportivo & $1993-$ & 22 & 90 & $24,4 \%$ \\
\hline Journal of Human Sport and Exercise & 2006- & 17 & 225 & $7,6 \%$ \\
\hline Archivos de medicina del deporte & $1984-$ & 16 & 336 & $4,8 \%$ \\
\hline Cultura, Ciencia y Deporte & 2004- & 16 & 136 & $11,8 \%$ \\
\hline RICYDE. Revista Internacional de Ciencias del Deporte & $2005-$ & 15 & 170 & $8,8 \%$ \\
\hline Apunts medicina de l'esport & $1964-$ & 14 & 220 & $6,4 \%$ \\
\hline Retos & $2002-$ & 14 & 237 & $5,9 \%$ \\
\hline Selección & $1989-2007$ & 14 & 158 & $8,9 \%$ \\
\hline $\begin{array}{l}\text { Revista internacional de medicina y ciencias de la actividad } \\
\text { física y del deporte }\end{array}$ & $2000-$ & 12 & 330 & $3,6 \%$ \\
\hline Revista iberoamericana de psicología del ejercicio y el deporte & 2006- & 10 & 108 & $9,3 \%$ \\
\hline Revista Internacional de Deportes Colectivos & $2008-$ & 9 & 57 & $15,8 \%$ \\
\hline Revista internacional de derecho y gestión del deporte & $2008-$ & 9 & 85 & $10,6 \%$ \\
\hline Journal of Sport and Health Research & $2009-$ & 6 & 77 & $7,8 \%$ \\
\hline Movimiento Humano & 2011- & 6 & 25 & $24 \%$ \\
\hline Revista Andaluza de Medicina del Deporte & $2008-$ & 6 & 112 & $5,4 \%$ \\
\hline Acción Motriz & $2008-$ & 4 & 50 & $8 \%$ \\
\hline AGON & 2011- & 3 & 21 & $14,3 \%$ \\
\hline EmásF & $2009-$ & 3 & 128 & $2,3 \%$ \\
\hline Materiales para la Historia del Deporte & 2003- & 3 & 61 & $4,9 \%$ \\
\hline Revista de educación física & $1985-$ & 3 & 214 & $1,4 \%$ \\
\hline Wanceulen e.f. digital & $2005-$ & 3 & 95 & $3,2 \%$ \\
\hline e-balonmano.com. Revista de Ciencias del Deporte & $2005-$ & 2 & 93 & $2,2 \%$ \\
\hline Habilidad Motriz & $1992-$ & 2 & 123 & $1,6 \%$ \\
\hline Revista Española de Educación Física y Deportes & $1949-$ & 2 & 175 & $1,1 \%$ \\
\hline Actividad Física y Deporte: Ciencia y Profesión & $2001-$ & 1 & 66 & $1,5 \%$ \\
\hline Ágora para la educación física y el deporte & $2001-$ & 1 & 120 & $0,8 \%$ \\
\hline Aloma: Revista de Psicología, Ciències de l'Educació i de l'Esport & $1997-$ & 1 & 321 & $0,3 \%$ \\
\hline Citius, altius, fortius & $2008-$ & 1 & 57 & $1,8 \%$ \\
\hline TRANCES: Transmisión del conocimiento educativo y de la salud & $2009-$ & 1 & 133 & $0,8 \%$ \\
\hline $\begin{array}{l}\text { ATHLOS. Revista Internacional de Ciencias Sociales de la } \\
\text { Actividad Física, el Juego y el Deporte }\end{array}$ & 2011- & 0 & 20 & $0 \%$ \\
\hline Coaching and Sport Science Review & 1993- & 0 & 235 & $0 \%$ \\
\hline FEGUI. Revista en Salvamento Acuático y Primeros Auxilios & $1997-$ & 0 & 53 & $0 \%$ \\
\hline Revista de Artes Marciales Asiáticas & 2006- & 0 & 140 & $0 \%$ \\
\hline
\end{tabular}

Fuente: Elaboración propia.

El número de artículos de fútbol publicados por año refleja una clara tendencia ascendente, multiplicándose por seis durante el periodo estudiado. Esta misma tendencia se 
observa en el total de artículos publicados. El rango en que se mueve el porcentaje de artículos sobre fútbol publicados respecto al total oscila entre el 6 y el 10\%, excepto en los dos primeros años analizados que no llega al 5\% (Tabla 2).

Tabla 2 - Evolución temporal del número de artículos de fútbol publicados y porcentaje respecto al total de artículos

\begin{tabular}{cccc}
\hline Año & Artículos sobre fútbol & Artículos publicados & $\%$ \\
\hline 2001 & 12 & 272 & $4,41 \%$ \\
2002 & 17 & 347 & $4,89 \%$ \\
2003 & 22 & 329 & $6,68 \%$ \\
2004 & 26 & 368 & $7,06 \%$ \\
2005 & 31 & 457 & $6,78 \%$ \\
2006 & 32 & 479 & $6,68 \%$ \\
2007 & 45 & 511 & $8,8 \%$ \\
2008 & 42 & 557 & $7,54 \%$ \\
2009 & 69 & 721 & $9,57 \%$ \\
2010 & 60 & 826 & $7,26 \%$ \\
2011 & 75 & 926 & $8,09 \%$ \\
2012 & 71 & 938 & $7,56 \%$ \\
\hline
\end{tabular}

Fuente: Elaboración propia.

Por último, las disciplinas abordadas ascendieron a 20 (Tabla 3). Las disciplinas más destacadas fueron Psicología y Legislación. Por el contrario, 10 disciplinas (50\%) no alcanzaron un promedio de un artículo de fútbol por año.

Tabla 3 - Número de artículos de fútbol por disciplina

\begin{tabular}{|c|c|c|}
\hline Disciplinas & № de artículos & $\%$ \\
\hline Psicología & 101 & 20,12 \\
\hline Legislación & 90 & 17,93 \\
\hline Organización y Gestión & 47 & 9,36 \\
\hline Táctica y estrategia & 46 & 9,16 \\
\hline Fisiología & 45 & 8,96 \\
\hline Teoría del Entrenamiento Deportivo & 36 & 7,17 \\
\hline Medicina del Deporte & 34 & 6,77 \\
\hline Didáctica & 28 & 5,58 \\
\hline Sociología & 16 & 3,19 \\
\hline Antropometría & 14 & 2,8 \\
\hline Biomecánica & 9 & 1,79 \\
\hline Nutrición & 8 & 1,59 \\
\hline Documentación & 7 & 1,39 \\
\hline Historia & 6 & 1,2 \\
\hline Nuevas tecnologías & 4 & 0,8 \\
\hline Técnica & 4 & 0,8 \\
\hline Teoría de la Educación Física y el Deporte & 3 & 0,6 \\
\hline Reglamento de competición & 2 & 0,4 \\
\hline Antropología & 1 & 0,2 \\
\hline Ciencias de la Información & 1 & 0,2 \\
\hline
\end{tabular}




\section{DISCUSIÓN}

El presente estudio identificó y analizó desde una perspectiva bibliométrica los artículos sobre fútbol publicados en las revistas españolas de CCAFD entre los años 2001-2012, poniéndolos en contexto respecto al total de artículos publicados. En términos generales, la producción científica sobre fútbol ha sido creciente, multidisciplinar y dispar entre las diversas revistas y disciplinas que se han ocupado de este deporte, y supuso el 7,9\% del total de artículos publicados en estas revistas. Aunque no existen estudios similares sobre otros deportes, puede considerarse que el fútbol ha sido generador de una destacable producción científica en un ámbito, las CCAFD, en el que confluyen un gran número de campos de conocimiento y prácticas físico-deportivas (BIRRELL, 2006). Entre los motivos que pueden explicar esta alta producción pueden citarse: (1) La dimensión social y económica del fútbol como "deporte rey" en España e internacionalmente, siendo un ejemplo paradigmático del proceso de globalización de las sociedades contemporáneas (TIESLER; COELHO, 2008); (2) Su dimensión como práctica que abarca todas las vertientes del deporte, desde el ámbito más lúdico al deporte más profesionalizado (MEDINA, 2009); (3) Su presencia en el ámbito universitario; y (4) El desarrollo de la investigación en las CCAFD y el incremento de publicaciones científicas donde difundir la investigación (VALENCIANO et al., 2010).

La Tabla 1 muestra cómo el fútbol ha estado presente en la gran mayoría de revistas de CCAFD españolas, aunque de forma muy desigual. En términos absolutos destaca la Revista Aranzadi de Derecho del Deporte y Entretenimiento. Esta revista, sin estar relacionada con el estudio del deporte fútbol en su concepto más práctico, ha dedicado al fútbol buena parte de sus contenidos. Esto se debe a que el fútbol tiene una gran relevancia en sectores sociales, culturales y económicos (AZNAR; ESTRUCH, 2012), con consecuencias a nivel legal y económico sobre los derechos de jugadores, entrenadores y los propios clubes, aspectos que entran totalmente en el ámbito de la revista. Estas consideraciones también se ven apoyadas por los altos porcentajes de artículos sobre fútbol publicados en las otras dos revistas especializadas en derecho deportivo: Revista española de derecho deportivo $(24,4 \%)$ y Revista internacional de derecho y gestión del deporte $(10,6 \%)$.

A continuación, destacan la Revista de psicología del deportey Cuadernos de psicología del deporte, ambas especializadas en psicología, a las que acompaña la Revista iberoamericana de psicología del ejercicio y el deporte. En este ámbito aparecen numerosos artículos debido a su alta productividad (DEVÍS et al., 2010) y a que el fútbol puede proporcionar una actividad profesional bien remunerada económicamente y/o reconocida socialmente a los psicólogos. En este sentido Garcia-Naveira y Jerez (2012) destacan la aparición de numerosas actividades de especialización para el psicólogo del fútbol y sus continuas actualizaciones para la práctica profesional. Otro aspecto favorecedor, en comparación con otras prácticas deportivas, es que la popularidad del fútbol facilita conseguir muestras amplias de deportistas para la realización de estudios experimentales. Estas apreciaciones encuentran apoyo en los resultados del estudio de Arbinaga, Aragón y Tejedor (2010), que situaron al fútbol como el deporte sobre el que se publicaron más artículos en la Revista de psicología del deporte durante el periodo 1992-2009.

El cuarto lugar respecto al total de artículos de fútbol publicados lo ocupa Apunts. Educación Física y Deportes, una publicación que responde a la tipología de revista multidisciplinar de CCAFD. Como comentan Devís et al. (2010), esta revista publica un número 
considerable de artículos debido a su periodicidad trimestral y diversidad temática. Este tipo de revistas multidisciplinar es el mayoritario en España en las CCAFD, y estas presentan porcentajes muy dispares respecto a la publicación de artículos de fútbol que oscilan entre el $0,3 \%$ y el $14,3 \%$, y puede entenderse como consecuencia lógica de la tradición y líneas editoriales que sigue cada revista.

Por último, destaca la baja presencia de artículos de fútbol en revistas especializadas en medicina del deporte (Archivos de medicina del deporte, Apunts medicina de l'esport, Revista Andaluza de Medicina del Deporte) todas ellas por debajo de la media. Al igual que en el caso de la psicología, la medicina aplicada al fútbol puede ser una actividad profesional reconocida y bien remunerada, por lo que parece contradictorio que no se hayan publicado un mayor número de artículos sobre fútbol en estas revistas. Aparte de otros motivos, el estudio de disciplinas (Tabla 3) muestra a la medicina del deporte en séptimo lugar (6,77\% de artículos), esto indica que parte de los estudios realizados en este ámbito son publicados en otras revistas menos específicas.

Respecto a la evolución temporal del número de artículos de fútbol, esta es evidente, hasta multiplicarse por seis durante el periodo estudiado. Según muestran los porcentajes relativos, este incremento se asocia más al incremento de revistas de CCAFD (un 62,5\% de los títulos analizados nacieron durante el periodo estudiado), que al incremento relativo de la investigación sobre fútbol sobre otros ámbitos, el cual también existe pero a una escala mucho menor. En esta línea, Devís et al. (2010) apuntan el notable crecimiento de la producción científica de las CCAFD que viene aparejado con la consolidación de esta comunidad académica en la universidad española. Asimismo, la amplia presencia de artículos de fútbol se puede explicar porque el fútbol ha sido un deporte muy popular durante todo el periodo estudiado (GARCÍA; LLOPIS, 2011), y no un deporte de moda generador de producción científica tan solo puntualmente.

Por su parte, en nuestro trabajo la disciplina más productiva fue la psicología. La circunstancia que más ha podido contribuir a ello es la existencia de tres revistas especializadas en psicología del deporte (todas ellas incluidas actualmente en bases de datos altamente reconocidas a nivel internacional, e.g., WoS y/o Scopus), lo cual favorece el desarrollo de este ámbito de conocimiento a través de temáticas como intervenciones psicológicas para la mejora de la concentración, motivación, estrés y ansiedad, estado de ánimo o liderazgo en el fútbol (SALVADOR et al., 2007). Asimismo, como apuntan Mora, Zarco y Blanca (2001), en psicología del deporte se da un creciente interés hacia los temas monográficos (como pueda ser el fútbol), en lugar de hacia los grandes sistemas conceptuales de la psicología deportiva. En el mismo sentido, la existencia de tres revistas especializadas en los ámbitos de legislación y organización del deporte explica que la aportación del conjunto de estas disciplinas (legislación y organización y gestión del deporte) sume la mayor producción científica hallada en este trabajo.

En tercer lugar, la táctica y la estrategia se muestra como una de las disciplinas más destacadas dentro del estudio del fútbol por su importancia para la mejora del rendimiento y su tradición en el espacio profesional y educativo de los deportes colectivos. Según García, Rodríguez y Garzón (2011) el fútbol es un deporte táctico, donde el termino inteligencia táctica se asocia a talento y al éxito deportivo. García (2008), en la misma línea, destaca la importancia de aspectos tratados por la táctica, como son las posibilidades de desarrollo de la inteligencia 
de juego, las relaciones que se establecen entre conocimiento procedimental y toma de decisiones, y la búsqueda de unas fases sensibles en la formación táctica del futbolista.

También ha de señalarse la importante producción de artículos correspondientes a las disciplinas médicas, donde destacan la fisiología y la medicina del deporte, las cuales también enfatizan Devís et al. (2010) por su protagonismo en la evolución histórica del conocimiento científico de las CCAFD y en la formación de sus profesionales. Estas disciplinas se ven respaldadas por revistas especializadas que han formado parte de este estudio, como Archivos de medicina del deporte, Apunts medicina de l'esport, y Revista Andaluza de Medicina del Deporte, pero también por otras genéricas de las CCAFD que conceden bastante importancia a estas disciplinas, como por ejemplo la Revista Internacional de Medicina y Ciencias de la Actividad Física y el Deporte.

El sexto lugar en nuestro estudio lo ocupa la teoría del entrenamiento deportivo, un ámbito en constante evolución que en la actualidad tiende a considerar el entrenamiento como un proceso complejo y estructurado orientado hacia el éxito deportivo (SEIRUL-LO, 2005). Dentro de este ámbito, la preparación física de los futbolistas es una de las tareas más valoradas y solicitadas en el fútbol de competición, por su alta demanda de capacidades físicas como fuerza, resistencia y velocidad, y los largos periodos de competición que tienen que afrontar los atletas. Esto hace que la preparación física de los futbolistas sea un proceso determinante (GÓMEZ, 2011), lo cual ha despertado el interés de la comunidad científica y una elevada producción de artículos.

A continuación, la didáctica y la pedagogía también destacan como disciplinas relevantes en el ámbito de las CCAFD en general (DEVÍS et al., 2010) y del fútbol en particular. Los avances en estas disciplinas han supuesto muchos cambios en la forma de entender las complejidades del fútbol, evolucionando desde modelos de enseñanza propios de deportes individuales, cuya relación con los deportes colectivos como el fútbol era mínima, a otros más específicos y coherentes con las características estructurales y funcionales de los deportes colectivos (ARJOL, 2012, CASARIN et al., 2011, STOLZ; PILL, 2014). Toda esta evolución ha generado una abundante literatura científica que también tiene su traducción en nuestro estudio.

\section{FUTURAS LÍNEAS DE INVESTIGACIÓN}

Futuras líneas de investigación podrían reproducir y comparar los resultados obtenidos en el presente estudio con otros deportes populares en España, como el baloncesto, el ciclismo o la natación. Igualmente, aplicarlo en un contexto geográfico más amplio para estudiar las similitudes y diferencias existentes en la evolución y tendencias de investigación sobre fútbol que se siguen actualmente a nivel internacional, y particularmente en países como Brasil, Francia, Reino Unido, etc., donde el fútbol es también considerado como el deporte rey.

\section{REFERENCIAS}

ARBINAGA, Félix; ARAGÓN, Juan Diego; TEJEDOR, Rocío. Análisis bibliométrico de la Revista de Psicología del Deporte (1992-2009). Revista de Psicología del Deporte, Palma de Mallorca, 
ARJOL, José Luis. La planificación actual del entrenamiento en fútbol: análisis comparado del enfoque estructurado y la periodización táctica. Acción Motriz, Las Palmas de Gran Canaria, n. 8, p. 27-37, 2012.

AZNAR, Jerónimo; ESTRUCH, Vicente. Determinación del valor de traspaso de jugadores de fútbol profesional. Valoración multicriterio. Revista Internacional de Derecho y Gestión del Deporte, Madrid, n. 17, p. 3-22, 2012.

BIRRELL, Susan. Sport and Sport Studies. In: ROJEK, C.; SHAW, S; VEAL, T. (Eds.). The handbook of leisure studies. London: Palgrave Macmillan, 2006. p. 335-353.

CASARIN, Rodrigo Vicenzi et al. Modelo de jogo e processo de ensino no futebol: princípios globais e específicos. Movimento, Porto Alegre, v. 17, n. 3, p. 133-152, 2011.

CIOMAGA, Bogdan. Sport management: a bibliometric study on central themes and trends. European Sport Management Quarterly, London, v. 13, n. 5, p. 557-578, 2013.

CONSEJO SUPERIOR DE DEPORTES. Memoria 2013: Licencias y Clubes. 2014. Disponible en: <http://www.csd.gob.es/csd/estaticos/asoc-fed/licenciasyclubes-2013.pdf>. Acceso en: 18 ago. 2014.

DEVÍS, José et al. Disciplinas y temas de estudio en las ciencias de la actividad física y el deporte. Revista Internacional de Medicina y Ciencias de la Actividad Física y del Deporte, Madrid, v. 10, n. 37 , p. 150-166, 2010.

DEVÍS, José Devís et al. Las revistas científico-técnicas españolas de las ciencias de la actividad física y el deporte: inventario y análisis de la calidad de contenido y difusión. Revista Española de Documentación Científica, Madrid, v. 26, n. 2, p. 177-190, 2003.

FERNÁNDEZ-REVELLES, Andrés B. ABFR-INDEX: correlación entre producción científica en "fútbol" y ranking. Revista Internacional de Medicina y Ciencias de la Actividad Física y del Deporte, Madrid, v. 14, n. 56, p. 705-718, 2014.

FÉDÉRATION INTERNATIONALE DE FOOTBALL ASSOCIATION. FIFA Big Count 2006: 270 million people active in football. 2007. Disponible en: $<$ http://www.fifa.com $/ \mathrm{mm} /$ document/fifafacts/ bcoffsurv/bigcount.statspackage_7024.pdf>. Acceso en: 29 sept. 2014.

FÉDÉRATION INTERNATIONALE DE FOOTBALL ASSOCIATION. The 2014 FIFA World Cup in Numbers. 2014. Disponible en: <http://resources.fifa.com/mm/document/tournament/ competition/02/44/29/89/fifaworldcupinnumbers 120714_v7 eng neutral.pdf>. Acceso en: 29 sept. 2014.

GARCÍA, Luis Miguel. Investigación y enseñanza técnico-táctica en el fútbol. Cultura Ciencia y Deporte, Murcia, v. 9, n. 3, p. 161-168, 2008.

GARCÍA, Manuel; LLOPIS, Ramón. Encuesta sobre los hábitos deportivos en España 2010: ideal democrático y bienestar personal. Madrid: CSD/CIS, 2011.

GARCÍA, Sandra; RODRÍGUEZ, Andrés; GARZÓN, Alexandra. Conceptualización de inteligencia táctica en fútbol: consideraciones para el desarrollo de un instrumento de evaluación en campo desde las funciones ejecutivas. Cuadernos de Psicología del Deporte, Murcia, v. 11, n. 1, p. 69-78, 2011.

GARCÍA-NAVEIRA, Alejo; JEREZ, Pilar. Departamento de psicología del club Atlético de Madrid: filosofía, programación y desempeño profesional en el fútbol base. Cuadernos de Psicología del deporte, Murcia, v. 12 , n. 1, p. 111-120, 2012. 
GAU, Li-Shiue. Trends and topics in sports research in the Social Science Citation Index from 1993 to 2008. Perceptual \& Motor Skills, Missoula, v. 116, n. 1, p. 305-314, 2013.

GÓMEZ, Pedro. La preparación física en el fútbol contextualizada en el fútbol. Pontevedra: MC Sports, 2011.

GUTIÉRREZ, Óscar. Análisis de la producción científica en balonmano en las revistas de la web of science. e-balonmano.com: Revista de Ciencias del Deporte, Mérida, v. 10, n. 2, p. 77-88, 2014.

KAUR, Jasleen; RADICCHI, Filippo; MENCZER, Filippo. Universality of scholarly impact metrics. Journal of Informetrics, Amsterdam, v. 7, n. 4, p. 924-932, 2013.

LIDOR, Ronnie; MILLER, Uri; MAHARIK, Ayala. Bibliometric analysis in sport and physical education sciences: theoretical background and research implications. In: GHENT, Gretchen. (Ed.). Sport and information technology. Oxford: Meyer \& Meyer Sport, 2002. p. 95-103.

LÓPEZ, Alberto; VELASCO, Alfonso. Breve análisis de la producción científica de la revista Archivos de medicina del deporte: periodo 1984-2002. Archivos de Medicina del Deporte, Pamplona, v. 21, n. 100, p. 93-98, 2004.

MARTíN, Ignacio et al. Bibliometric study (1922-2009) on rugby articles in research journals. South African Journal for Research in Sport, Physical Education \& Recreation, Stellenbosch, v. 35, n. 1, p. 105-119, 2013.

MARTíNEZ, Julio A. et al. Producción científica sobre cardiología y deporte: ranking de revistas (2010) y excelencia. Revista Internacional de Medicina y Ciencias de la Actividad Física y del Deporte, Madrid, v. 12, n. 46, p. 299-312, 2012.

MARTíNEZ, Julio A. et al. (2002). Análisis documental y bibliométrico sobre el arbitraje en el fútbol en la base de datos sportdiscus. 1830-2002. In: JORNADAS CANARIAS DE TRAUMATOLOGÍA Y CIRUGÍA ORTOPÉDICA PARA ESPECIALISTAS Y POST-GRADUADOS, 16., 2002, Las Palmas de Gran Canaria. Libro de actas... Las Palmas de Gran Canaria: Hospital Insular, 2002. p. 199-200.

MCBRIDE, Ron E. Impact factors for the Journal of Teaching in Physical Education - what are they and are they important? Journal of Teaching in Physical Education, Champaign, v. 25, n. 1, p. 3-8, 2006.

MEDINA, F. El fútbol y la vivencia festiva de la nacionalidad. Razón y palabra, México, n. 69, 2009. Disponible en: <www.razonypalabra.org.mx>. Acceso en: 26 abr. 2014.

MORA, Juan Antonio; ZARCO, Juan Antonio; BLANCA, María José. Atención - Concentración como entrenamiento para la mejora del rendimiento deportivo en jugadores profesionales de fútbol. Revista de Psicología del Deporte, Palma de Mallorca, v. 10 , n. 1, p. 49-65, 2001.

NAVARRO-GARCÍA, Antonio; REYES-GARCÍA, María Eugenia; ACEDO-GONZÁLEZ, Francisco Javier. Calidad percibida y satisfacción de los espectadores de fútbol. Investigaciones Europeas de Dirección y Economía de la empresa, Vigo, v. 20, n. 2, p. 87-94, 2014.

NEVILL, Alan; ATKINSON, Greg; HUGHES, Mike. Twenty-five years of sport performance research in the Journal of Sports Sciences. Journal of Sports Sciences, London, v. 26 , n. 4, p. 413-426, 2008. 
PÉREZ-GUTIÉRREZ, Mikel et al. The (Re)Emergence of a Religio-Spiritual Self-Cultivation Focus in Asian Martial Arts Monographs Published in Spain (1906-2009). The International Journal of the History of Sport, London, v. 32, n. 2, p. 200-217, 2015.

PÉREZ-GUTIÉRREZ, Mikel; GUTIÉRREZ-GARCÍA, Carlos. Estudio bibliométrico sobre las monografías de artes marciales publicadas en España (1906-2006). Revista de Artes Marciales Asiáticas, León, v. 3, n. 4, p. 22-33, 2008.

PÉREZ-GUTIÉRREZ, Mikel; GUTIÉRREZ-GARCÍA, Carlos. Bibliometric analysis of karate monographs in Spain (1963-2006). In: CYNARSKI, Wojciech Jan. (Ed.). Martial Arts and Combat Sports - Humanistic Outlook. Rzeszow: Wydawnictwo universytetu Rzeszowskiego, 2009. p. 116-126.

PESET, Fernanda. et al. Scientific literature analysis of Judo in Web of Science. Archives of Budo, Warsaw, v. 9, n. 2, p. 81-91, 2013.

PIERNAVIEJA, Miguel. El libro deportivo español. Citius, Altius, Fortius, Madrid, v. 7, n. 1, p. 91-115, 1965.

SALVADOR, Simone et al. Análise da produção científica em Psicologia do Esporte. Revista Iberoamericana de Psicología del Ejercicio y el Deporte, Las Palmas de Gran Canaria, v. 2, n. 1, p. 25-40, 2007.

SÁNCHEZ, José Manuel; CASTELLANOS, Pablo. A Bibliometric Analysis of Sports Economics Research. International Journal of Sport Finance, Morgantown, v. 6, n. 3, p. 222-244, 2011.

SÁNCHEZ, José Manuel; CASTELLANOS, Pablo. La economía del deporte en España, análisis bibliométrico de una década (2002-2011). Estudios de Economía Aplicada, Valladolid, v. 30, n. 2, 419-439, 2012.

SEIRU-LO, Francisco. Línea general de trabajo para el fútbol como deporte de equipo. Training Fútbol, Valladolid, n. 109, p. 26-33, 2005.

STOLZ, Steven; PILL, Shane. Teaching games and sport for understanding: Exploring and reconsidering its relevance in physical education. European Physical Education Review, London, v. 20, n. 1, p. 36-71, 2014.

TIESLER, Nina Clara; COELHO, João Nuno (Eds). Globalised football: nations and migration, the city and the dream. New York: Routledge, 2008.

USING bibliometrics: A guide to evaluating research performance with citation data. Thomson Reuters, 2008. Disponible en: <http://ip-science.thomsonreuters.com/m/pdfs $/ 325133$ thomson.pdf $>$. Acceso en: 27 ene. 2015.

VALENCIANO, Javier et al. La colaboración científica en el campo de las Ciencias de la actividad física y el deporte en España. Revista Española de Documentación Científica, Madrid, v. 33, n. 1, p. 90-105, 2010.

VALENCIANO, Javier; DEVÍS, José; VILLAMÓN, Miguel. Autoría institucional de los trabajos publicados en revistas españolas de ciencias del deporte (1999-2005). Cultura, Ciencia y Deporte, Murcia, v. 4, n. 10, p. 7-14, 2009.

VILLAMÓN, Miguel; DEVIIS, José; VALENCIANO, Javier. Análisis de la visibilidad de las revistas científico-técnicas españolas de ciencias de la actividad física y el Deporte. Revista de Psicología del Deporte, Palma de Mallorca, v. 14, n. 2, p. 253-267, 2005. 
VILLAREJO, Diego; PALAO, Juan Manuel; ORTEGA, Enrique. La producción científica en Rugby Union entre 1998-2007. e-balonmano.com: Revista de Ciencias del Deporte, Mérida, v. 6, n. 3, p. 155-161, 2010. 